\title{
Severe bilateral cerebellar edema from ingestion of ketamine: case report
}

\author{
Nicolas Villelli, MD, ${ }^{1}$ Natalie Hauser, NP, ${ }^{1}$ Thomas Gianaris, MD, ${ }^{1}$ Blake A. Froberg, MD, ${ }^{2}$ and \\ Daniel H. Fulkerson, MD'
}

1Department of Neurological Surgery, Division of Pediatric Neurosurgery, Goodman Campbell Brain and Spine; and ${ }^{2}$ Department of Clinical Emergency Medicine and Pediatrics, Indiana University School of Medicine, Indianapolis, Indiana

\begin{abstract}
The use of ketamine as a drug of abuse has increased and so too has the risk of accidental overdose. Here, the authors report the case of a 10-month-old infant who inadvertently ingested ketamine. The child demonstrated severe cerebellar swelling that required emergency surgical intervention. The authors describe the clinical course of this child and present the radiographic characteristics of the brain. The imaging characteristics were not consistent with purely anoxic injury, thus suggesting a specific effect of this drug. To the authors' knowledge, similar imaging characteristics in this context have not been described.
\end{abstract}

https://thejns.org/doi/abs/10.3171/2017.5.PEDS16695

KEY WORDS ketamine; cerebellar; edema; pediatric neurosurgery

$\mathrm{A}$ NOXIC brain injury primarily affects the supratentorial regions of the brain. ${ }^{5,6}$ Often, the brainstem and cerebellar hemispheres are relatively spared from an anoxic injury. ${ }^{6}$ The sparing of the cerebellum creates the phenomenon known as the reverse cerebellum sign, in which the normal cerebellum appears relatively bright in comparison with the diffuse hypodensity of the cerebral hemispheres on CT scanning. ${ }^{3}$

Ketamine is a phencyclidine derivative and a noncompetitive antagonist of $N$-methyl-D-aspartate. ${ }^{9}$ It is a dissociative anesthetic used in human and veterinary medicine. Ketamine is also used to treat a number of psychiatric problems, including depression, bipolar disorder, anxiety, and chronic pain..$^{13,16,19}$ Ketamine has reinforcing and rewarding properties, leading to a potential for dependence. It is an increasingly popular "rave" drug. Ketamine abuse has increased significantly in the United States, the United Kingdom, and many countries in Asia. In addition to the psychotropic effects, ketamine affects the urinary system, liver, and cardiovascular system; it increases heart rate and blood pressure due to decreased catecholamine reuptake. ${ }^{10}$ Ketamine is also associated with pulmonary complications, including respiratory depression and apnea.

We describe the unique case of a 10-month-old infant who inadvertently ingested ketamine. The patient had se- vere bilateral cerebellar cytotoxic edema and swelling that caused obstructive hydrocephalus. The imaging characteristics were not consistent with purely anoxic injury and thus suggest a specific effect of the drug.

\section{Case Report}

A previously healthy 10-month-old girl was evaluated at an outside hospital after being found unresponsive by a family member. The patient's brother and grandmother were also found unresponsive. All 3 patients were initially suspected to be suffering from carbon monoxide poisoning. The 10-month-old infant was in critical condition with cyanosis and poor respiratory effort. She underwent emergency intubation.

The circumstances of the incident were initially unknown. A police investigation showed that family members were producing a drug of abuse in the house composed of tobacco leaves soaked in ketamine (called "Kommon"). The child was served eggs that were cooked in the same dishes used to prepare the drug. The child vomited after eating the eggs and then went back to bed. The other 2 family members also ate eggs prepared with the same dishes.

The patient was transferred on an emergency basis to 
Riley Hospital for Children, where she was noted to have a Glasgow Coma Scale score of 6T. She was withdrawing her left upper extremity, extensor posturing in her right upper extremity, and withdrawing her lower extremities bilaterally. Her lower extremities demonstrated increased muscular tone. Her pupils were $3 \mathrm{~mm}$ and sluggishly reactive.

The patient's initial laboratory findings showed a serum glucose level of $400 \mathrm{mg} / \mathrm{dl}$, sodium bicarbonate level of $8 \mathrm{mmol} / \mathrm{L}, \mathrm{PCO}_{2}$ greater than $52 \mathrm{~mm} \mathrm{Hg}$, and a base excess of $-20 \mathrm{mmol} / \mathrm{L}$. The carbon monoxide percentages were normal. Serum salicylate, acetaminophen, and ethanol concentrations were undetectable. A urine Drugs of Abuse Screen (enzyme immunoassay at Indiana University Health Pathology Laboratory), performed 16 hours after the initial presentation, was positive for benzodiazepines (the child received midazolam for sedation 12 hours after presentation) and negative for amphetamines, barbiturates, cannabinoids, cocaine, opiates, phencyclidine, and oxycodone. A prescription/over-the-counter urine drug screen (gas chromatography-mass spectrometry at Mayo Medical Laboratories), performed 20 hours after presentation, was positive for ketamine and levetiracetam (the child received levetiracetam 11 hours after presentation). A urine ketamine and metabolite confirmation test (gas chromatography-mass spectrometry at Mayo Medical Laboratories) detected ketamine $(271 \mathrm{ng} / \mathrm{ml})$ and norketamine $(847 \mathrm{ng} / \mathrm{ml})$. At no time during the child's admission was she given ketamine or a ketamine derivative for medical care. The ketamine levels were not available at time points more than 48 hours after admission.

Imaging studies performed when the child arrived at our institution revealed cytotoxic edema involving the bilateral cerebellar hemispheres with sparing of the superior vermis (Fig. 1A-C). There were localized foci of decreased diffusion within the right occipital lobe and the left hippocampal cortex, and within the bilateral periventricular white matter, with sparing of the globi pallidi. The swelling of the cerebellum obstructed the foramen magnum, effaced the fourth ventricle, and caused obstructive hydrocephalus (Fig. 1D). The patient underwent CT angiography and CT venography. All vessels in the anterior and posterior circulation showed normal anatomy and normal patency. There was no evidence of venous thrombosis or venous occlusion.

The patient underwent emergency placement of an external ventricular drain (EVD) and was taken for surgical decompression of the posterior fossa. The opening pressure of the EVD was approximately $20 \mathrm{~cm} \mathrm{H}_{2} \mathrm{O}$. A wide suboccipital craniotomy was performed, which extended to the lateral border of the foramen magnum inferiorly, approximately $2 \mathrm{~cm}$ lateral to the foramen magnum on either side, and $2 \mathrm{~cm}$ superiorly. The bone was not replaced.

After surgery, the patient remained intubated with a $\mathrm{PCO}_{2}$ goal of $40-45 \mathrm{~mm} \mathrm{Hg}$ and a mean arterial pressure goal of $50 \mathrm{~mm} \mathrm{Hg}$. She did not require ionotropic support. Her head was elevated to $30^{\circ}$, and hypertonic saline was administered intermittently for hyponatremia. The patient was placed on a regimen of levetiracetam therapy after electroencephalography showed seizure activity. The patient's neurological examination findings slowly improved;
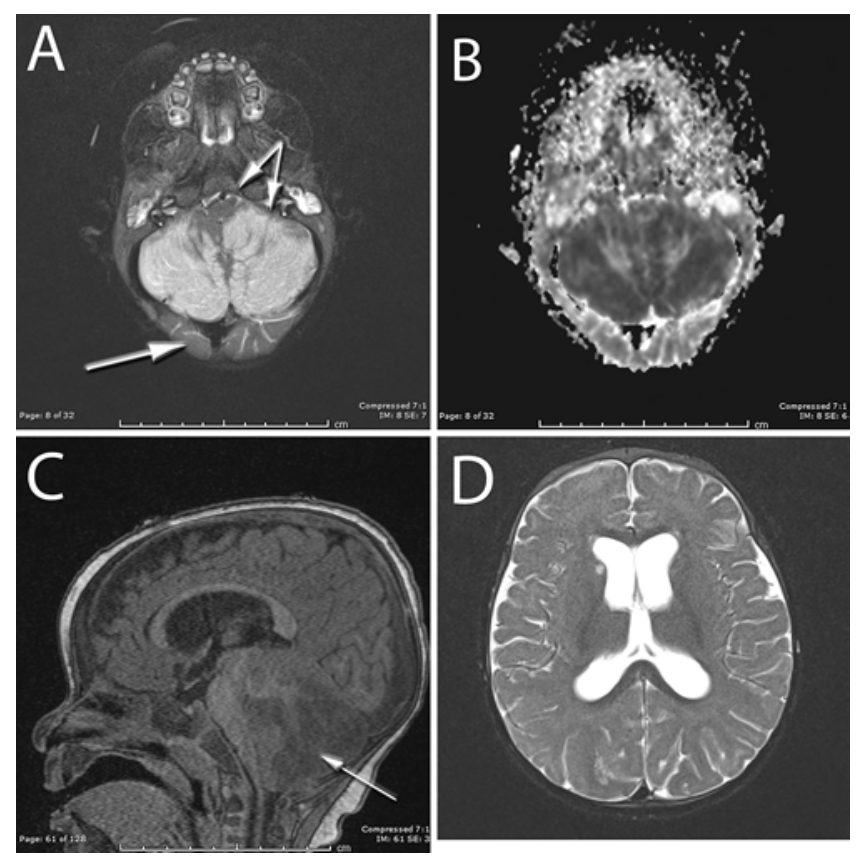

FIG. 1. A: Axial T2-weighted MR image showing hyperintensity that is indicative of cytotoxic edema in the bilateral cerebellar hemispheres. A slice that shows the comparison signal of the supratentorial occipital lobe is demonstrated (arrow). The double arrows highlight the difference in signal intensity between the compressed brainstem and the cerebellar hemispheres. B: Axial diffusion-weighted MR image demonstrating bilateral cerebellar infarctions (see text for more information). C: Sagittal noncontrast T1-weighted MR image showing significant cerebellar edema (arrow). D: Axial T2-weighted MR image demonstrating obstructive hydrocephalus with a hyperintensity in the periventricular caudate nucleus.

by postoperative Day 6 , the patient was extubated and was purposeful with all 4 extremities. A percutaneous endoscopic gastrostomy (PEG) tube was placed. We attempted to wean the patient off external ventricular drainage; however, the patient continued to have a high CSF output (> $200 \mathrm{ml}$ per day with the EVD set open at $20 \mathrm{~cm} \mathrm{H}_{2} \mathrm{O}$ ). We considered performing an endoscopic third ventriculostomy; however, we were concerned about the posterior fossa swelling thereby compressing the prepontine cistern (Fig. 1C). By 2 weeks, the patient had improved enough to participate in rehabilitation efforts. Her improvement continued, and 15 days after admission we decided to remove the EVD and place a CSF shunt.

The patient improved significantly and was discharged to an inpatient rehabilitation unit. Imaging 6 months after her presentation showed bilateral cerebellar hemisphere encephalomalacia (Fig. 2).

Clinically, the child made a remarkable recovery. One year after her presentation, she was ambulatory, moved all extremities symmetrically, and had no spasticity. She has exhibited a mild speech delay, but otherwise has met all age-appropriate developmental milestones. The PEG tube has been removed and she is able to tolerate a regular diet.

\section{Discussion}

Ketamine abuse is increasing because of its popularity 


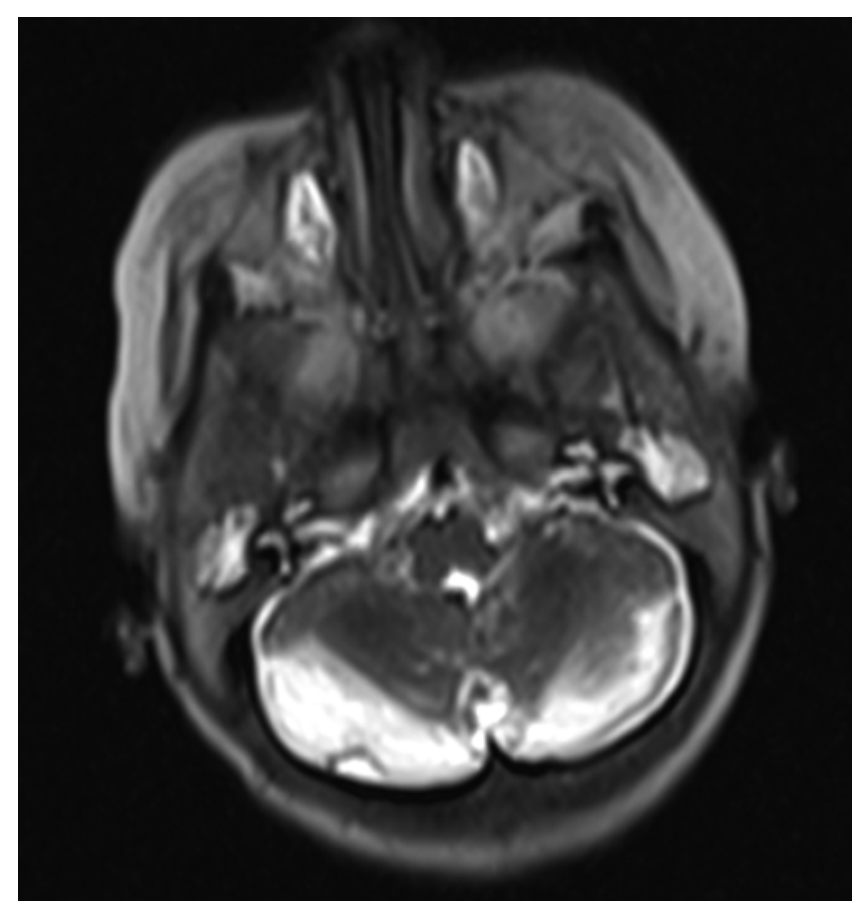

FIG. 2. Axial T2-weighted MR image obtained 6 months after the initial presentation, showing bilateral cerebellar encephalomalacia.

as a recreational party drug that induces relaxation and euphoria. The rise in uncontrolled use has led to increases in injury related to ketamine toxicity. In the United Kingdom, the number of ketamine-related deaths increased by 10 times from 1999 to 2008.11

Very few studies have evaluated the brain-imaging characteristics of children exposed to ketamine, and to our knowledge no reports have specifically described the effects on the brain from an overdose of this drug of abuse. Wang et al. analyzed brain MRI characteristics in adult ketamine addicts. Patients showed diffuse atrophy of the cerebral hemispheres, with sparing of the cerebellum and brainstem. ${ }^{17}$ The clinical characteristics of pediatric patients inadvertently exposed to an overdose of ketamine as an anesthetic have been described by Green et al. They observed prolonged sedation without any signs of neurological injury on follow-up examination. ${ }^{2}$ These authors did not report any cranial imaging results in their study. The remaining available data on ketamine exposure were obtained mainly in rat models. Multiple studies in models of infant rats have shown that ketamine has a neurodegenerative effect when doses reach the $20-\mathrm{mg} / \mathrm{kg}$ threshold and that the severity of degeneration correlates with higher doses. ${ }^{4,15}$

Our patient was found in respiratory distress, and it is reasonable to assume that she was at high risk for anoxic brain injury. However, her imaging studies did not show the classic anoxic brain injury pattern of findings in the cerebral hemispheres and basal ganglia. ${ }^{5,6}$ Rather than exhibiting the reverse cerebellum sign associated with anoxic injury, our patient's images revealed extensive anoxic injury in the cerebellar hemispheres, with near-complete sparing of the cerebral cortex and basal ganglia. Another unusual finding was that edema seen on the initial images exhibited an increased signal on diffusion-weighted sequencing and a decreased signal on apparent diffusion coefficient maps compared with normal brain parenchyma. Because the apparent diffusion coefficient map value is used to differentiate vasogenic from cytotoxic edema, we measured this value in a representative region of interest in the cerebellum. The resulting apparent diffusion coefficient map value of $474 \times 10^{-6} \mathrm{~mm}^{2} / \mathrm{sec}$ was within the accepted threshold of $\leq 620 \times 10^{-6} \mathrm{~mm}^{2} / \mathrm{sec},{ }^{12}$ indicating cytotoxic edema rather than vasogenic edema, for which values are higher. The combined findings in this patient were therefore consistent with cytotoxic edema?

Ketamine increases global cerebral blood flow and regional cerebral blood flow via vasodilation of intracranial vessels through a calcium-dependent mechanism. ${ }^{18}$ In a study of healthy adult male volunteers, Långsjö et al. demonstrated concentration-dependent increases in regional cerebral blood flow in the anterior cingulate, thalamus, putamen, and frontal cortex. These investigators also observed a subtle increase in the regional metabolic rate of oxygen in the frontal, parietal, and occipital lobes and a decrease in the cerebellum. ${ }^{8}$ In the case we describe, the effect of the ketamine-based drug may have protected the supratentorial brain from the effect of anoxia. The unique imaging characteristics in our patient may also have resulted from an as-yet-unknown direct effect of the drug on the cerebellum. We cannot rule out exposure to a different toxin; however, the testing was reasonably complete and the other affected family members also tested positive for ketamine.

Given the rarity of posterior circulation strokes, the prognosis of this finding in the pediatric population is not well described. ${ }^{1,14}$ Rosman et al. performed a literature search of posterior circulation infarctions in the pediatric population; they found that over half of the patients made full recoveries. ${ }^{14}$ Our patient had an excellent recovery after emergency surgery and treatment of hydrocephalus. At 1 year after the incident, she was ambulatory without spasticity and meeting all developmental milestones except for a mild speech delay.

\section{Conclusions}

To our knowledge, the imaging findings of bilateral cerebral cytotoxic edema with relative sparing of the cerebral hemispheres resulting from inadvertent ingestion of ketamine have not been previously presented. As the incidence of ketamine abuse increases, so does the potential for toxic exposure. Thus familiarity with characteristic findings such as those reported here will become increasingly important so that ketamine toxicity can be quickly recognized and appropriately treated.

\section{Acknowledgments}

We thank Dr. Chang Ho for assistance in interpreting the imaging studies.

\section{References}

1. Chatkupt S, Epstein LG, Rappaport R, Koenigsberger MR: Cerebellar infarction in children. Pediatr Neurol 3:363-366, 1987 
2. Green SM, Clark R, Hostetler MA, Cohen M, Carlson D, Rothrock SG: Inadvertent ketamine overdose in children: clinical manifestations and outcome. Ann Emerg Med 34:492-497, 1999

3. Han BK, Towbin RB, De Courten-Myers G, McLaurin RL, Ball WS Jr: Reversal sign on CT: effect of anoxic/ischemic cerebral injury in children. AJR Am J Roentgenol 154:361368,1990

4. Hayashi H, Dikkes P, Soriano SG: Repeated administration of ketamine may lead to neuronal degeneration in the developing rat brain. Paediatr Anaesth 12:770-774, 2002

5. Huang BY, Castillo M: Hypoxic-ischemic brain injury: imaging findings from birth to adulthood. Radiographics 28:417-439, 617, 2008

6. Jung DE, Ritacco DG, Nordli DR, Koh S, Venkatesan C: Early anatomical injury patterns predict epilepsy in head cooled neonates with hypoxic-ischemic encephalopathy. Pediatr Neurol 53:135-140, 2015

7. Koch S, Rabinstein A, Falcone S, Forteza A: Diffusionweighted imaging shows cytotoxic and vasogenic edema in eclampsia. AJNR Am J Neuroradiol 22:1068-1070, 2001

8. Långsjö JW, Kaisti KK, Aalto S, Hinkka S, Aantaa R, Oikonen V, et al: Effects of subanesthetic doses of ketamine on regional cerebral blood flow, oxygen consumption, and blood volume in humans. Anesthesiology 99:614-623, 2003

9. Liu Y, Lin D, Wu B, Zhou W: Ketamine abuse potential and use disorder. Brain Res Bull 126:68-73, 2016

10. Mayberg TS, Lam AM, Matta BF, Domino KB, Winn HR: Ketamine does not increase cerebral blood flow velocity or intracranial pressure during isoflurane/nitrous oxide anesthesia in patients undergoing craniotomy. Anesth Analg 81:84-89, 1995

11. Morgan CJ, Curran HV: Ketamine use: a review. Addiction 107:27-38, 2012

12. Purushotham A, Campbell BC, Straka M, Mlynash M, Olivot JM, Bammer R, et al: Apparent diffusion coefficient threshold for delineation of ischemic core. Int J Stroke 10:348353,2015

13. Romeo B, Choucha W, Fossati P, Rotge JY: Meta-analysis of short- and mid-term efficacy of ketamine in unipolar and bipolar depression. Psychiatry Res 230:682-688, 2015
14. Rosman NP, Wu JK, Caplan LR: Cerebellar infarction in the young. Stroke 23:763-766, 1992

15. Scallet AC, Schmued LC, Slikker W Jr, Grunberg N, Faustino PJ, Davis H, et al: Developmental neurotoxicity of ketamine: morphometric confirmation, exposure parameters, and multiple fluorescent labeling of apoptotic neurons. Toxicol Sci 81:364-370, 2004

16. Sheehy KA, Muller EA, Lippold C, Nouraie M, Finkel JC, Quezado ZM: Subanesthetic ketamine infusions for the treatment of children and adolescents with chronic pain: a longitudinal study. BMC Pediatr 15:198, 2015

17. Wang C, Zheng D, Xu J, Lam W, Yew DT: Brain damages in ketamine addicts as revealed by magnetic resonance imaging. Front Neuroanat 7:23, 2013

18. Zeiler FA, Sader N, Gillman LM, Teitelbaum J, West M, Kazina CJ: The cerebrovascular response to ketamine: a systematic review of the animal and human literature. J Neurosurg Anesthesiol 28:123-140, 2016

19. Zgaia AO, Irimie A, Sandesc D, Vlad C, Lisencu C, Rogobete A, et al: The role of ketamine in the treatment of chronic cancer pain. Clujul Med 88:457-461, 2015

\section{Disclosures}

The authors report no conflict of interest concerning the materials or methods used in this study or the findings specified in this paper.

\section{Author Contributions}

Conception and design: Fulkerson, Villelli. Acquisition of data: Fulkerson, Hauser, Gianaris. Analysis and interpretation of data: Fulkerson. Drafting the article: all authors. Critically revising the article: Fulkerson, Froberg. Reviewed submitted version of manuscript: Fulkerson. Approved the final version of the manuscript on behalf of all authors: Fulkerson. Study supervision: Fulkerson.

\section{Correspondence}

Daniel H. Fulkerson, Indiana University School of Medicine/ Goodman Campbell Brain and Spine, Riley Hospital for Children, 702 Barnhill Dr. \#1134, Indianapolis, IN 46202-5200. email: dfulkers@iupui.edu. 